\title{
Estrés Académico en Alumnos Ingresantes a la Carrera de Odontología en la Universidad Nacional de Córdoba, Argentina
}

\author{
Academic Stress of Freshmen Students at the Faculty of \\ Dentistry Universidad Nacional de Cordoba, Argentina
}

\author{
Ivana Rodríguez'; Gabriel M. Fonseca² \& Guillermo Aramburú ${ }^{1,3}$
}

RODRÍGUEZ, I.; FONSECA, G. M. \& ARAMBURÚ, G. Estrés académico en alumnos ingresantes a la carrera de Odontología en la Universidad Nacional de Córdoba, Argentina. Int. J. Odontostomat., 14(4):639-647, 2020.

RESUMEN: El estrés es un estado de inestabilidad y angustia emocional que dificulta la capacidad para concentrarse y funcionar eficazmente de forma rutinaria. En el ámbito de la educación, el estrés académico cuenta con tres dimensiones: estresores, reacciones sobre la salud en general y los reguladores del estrés. Varios estudios han señalado que los estudiantes de carreras de la salud pueden estar expuestos a niveles de estrés más elevados que la población en general. El propósito de este estudio fue evaluar el estrés académico autopercibido en alumnos ingresantes a la Carrera de Odontología de la Universidad Nacional de Córdoba (Córdoba, Argentina). Se realizó un estudio descriptivo, donde la muestra estuvo conformada por 291 estudiantes. La información se recolectó a través del cuestionario autoadministrado Inventario Sisco del Estrés Académico (Barraza Macías, 2007a,b). El análisis de los datos se llevó a cabo empleando baremos específicos, y para la asociación entre variables se utilizaron las pruebas de Pearson y Anova, fijando el nivel de significación estadística en $p<0,05$. Una mayoría significativa de los alumnos $(98,3 \%)$ presentó estrés académico, con un $88,81 \%$ de los participantes inclinándose por las categorías de intensidad más altas de estrés. Se identificó una muy significativa correlación entre las dimensiones Estresores y Reacciones $(p<0,001)$ y entre Estresores y Estrategias $(p=$ 0,034). La identificación de estrés académico, su caracterización y la prevención respecto a la potencial instalación de hábitos o empleo de medicamentos en muchos casos insalubres, permitirá desarrollar estrategias que favorezcan a los estudiantes el poder hacer frente a las situaciones que el nuevo entorno demanda.

PALABRAS CLAVE: estrés autopercibido, estudiantes de odontología, estresor, estrategias de afrontamiento.

\section{INTRODUCCIÓN}

El estrés es un estado de inestabilidad y angustia emocional que dificulta la capacidad para concentrarse y funcionar eficazmente de forma rutinaria. Una persona desarrolla estrés debido a aspectos multifactoriales de la vida, pudiendo ser una preocupación irritante subconsciente o un estado de urgencia consciente que disminuye la eficiencia de trabajo y la productividad de las personas (Qamar et al., 2015). En el ámbito académico, cuando los requerimientos de una situación exceden los recursos y capacidades para adaptarse, el estudiante comienza a sentirse estresado. El estrés académico encierra tres dimensiones bien marcadas: la primera tiene en cuenta los estresores académicos; la segunda, los efectos del estrés académico sobre la salud en general; y la tercera dimensión engloba a los reguladores del estrés, tal es el caso de las características personales de cada estudiante (Rodríguez García et al., 2016; Quito Calle et al., 2017).

La respuesta al estrés comprende una amplia gama de modificaciones fisiológicas, metabólicas y neuroendocrinas. Entre ellas se destacan la activación del eje simpático-médulo-adrenal (SMA), que da lugar a cambios cardiovasculares y a la liberación de catecolaminas, y la activación del eje hipotalámico-

\footnotetext{
${ }^{1}$ Cátedra de Introducción a la Odontología, Facultad de Odontología, Universidad Nacional de Córdoba, Córdoba, Argentina.

${ }^{2}$ Centro de Investigación en Odontología Legal y Forense, Facultad de Odontología, Universidad de la Frontera, Temuco, Chile.

${ }^{3}$ Cátedra de Farmacología y Terapéutica B, Facultad de Odontología, Universidad Nacional de Córdoba, Córdoba, Argentina.
} 
hipofisario-adrenal (HHA), responsable de la liberación de corticosteroides al torrente sanguíneo (Nadal \& Armario, 2010).

Desde principios del s. XXI, la comunidad científica se debate entre dos enfoques principales para la aproximación y conocimiento de este fenómeno en el ámbito educativo: el enfoque psicobiológico en sus vertientes unidimensional y bidimensional, y el enfoque personológico cognitivista en su vertiente transaccional. El primero, identifica al estrés académico teniendo en cuenta los estresores y las manifestaciones psicobiológicas y se centra en la búsqueda de eventos estresores típicos del proceso de enseñanza y aprendizaje, como la falta de tiempo, la realización de exámenes y la obtención de resultados no satisfactorios, entre otros. Además, identifica manifestaciones fisiológicas, cognitivas y conductuales dentro de las que pueden describirse algunas como trastornos del sueño, digestivos, enfermedades psicosomáticas, aislamiento, pensamientos negativos, inmunodeficiencia, entre otros. El segundo enfoque, aborda el estrés desde una dimensión sistémica y cognitivista, donde los procesos cognitivos del individuo juegan el papel determinante en la aparición del estrés. Se reconoce como componente fundamental la evaluación que realice el individuo de la situación, de las demandas del entorno y de sus recursos individuales y sociales para considerarla desbordante o no (Marín Laredo et al., 2014)

En el modelo denominado Sistémico Cognoscitivista del estrés académico (Barraza Macías, 2007a), se definen como componentes estructurales a los estresores, la evaluación cognitiva, el afrontamiento y las manifestaciones percibidas a través de una interacción dinámica entre ellos y el entorno. La situación estresante se manifiesta en los alumnos a través de una serie de indicadores del desequilibrio, los cuales pueden ser clasificados como: físicos, psicológicos y comportamentales. Entre los físicos se encuentran aquellos que implican una reacción propia del cuerpo, como la onicofagia, temblores musculares, migrañas, insomnio, problemas de digestión y somnolencia, entre otros. Los psicológicos son aquellos relacionados con las funciones cognoscitivas o emocionales de la persona, como la inquietud, problemas de concentración, depresión, ansiedad, entre otros. Entre los comportamentales se describen aquellos que involucran la conducta de la persona, como iniciar discusiones repentinas, aislamiento de los demás, aumento o reducción del consumo de alimentos y desgano para realizar las labores escolares, entre otros (Berrío García \& Mazo Zea, 2011).
Algunas actividades académicas que pueden considerarse como propias de estrés académico son la realización de exámenes, exposición de trabajos en clases, intervención en el aula, sobrecarga académica, masificación de las aulas, competitividad entre compañeros, trabajar en grupo, exposiciones orales, la puntualidad, las exigencias del profesor, entre otros. Las consecuencias de los altos niveles de estrés que experimentan los estudiantes que van desde estados depresivos, ansiedad, irritabilidad, descenso de la autoestima, insomnio, asma, hipertensión, úlceras, etc., llegan a afectar tanto la salud como el rendimiento académico de los alumnos (Barraza Macías, 2005; Barraza Macías \& Silerio Quiñónez, 2007).

Existen numerosos trabajos que indican que los estudiantes de las áreas referidas a ciencias de la salud muestran niveles de estrés académico significativamente mayores a los de sus iguales que cursan otras disciplinas (Cabanach et al., 2016; Castillo Pimienta et al., 2016). Esto trae consigo la necesidad de que adopten estrategias de afrontamiento distintas de las que disponían hasta el momento, de manera que puedan superar las nuevas exigencias. Sin embargo, una gran parte de los estudiantes, carecen de esas estrategias o presentan conductas académicas inadecuadas para las nuevas demandas (Díaz Cárdenas et al., 2014). Además, se sabe que el estilo de vida de los estudiantes se ve modificado según se acerca el período de exámenes, convirtiendo sus hábitos en insalubres (exceso en el consumo de cafeína, tabaco, sustancias psicoactivas como excitantes e incluso ingesta de tranquilizantes), lo que a la larga puede llevar a la aparición de trastornos en la salud tanto física como mental (Martín Monzón, 2007). El propósito de este trabajo fue, por lo tanto, determinar el nivel de estrés académico autopercibido y caracterizar el mismo, en una muestra de alumnos ingresantes a la Facultad de Odontología de la Universidad Nacional de Córdoba en la ciudad de Córdoba, Argentina.

\section{MATERIAL Y MÉTODO}

Se llevó a cabo un estudio de tipo descriptivo y de corte transversal.

Población y muestra: El universo de estudio estuvo constituido por todos los alumnos matriculados (985) para el cursado del Ciclo Introductorio de Nivelación de la Facultad de Odontología (CINFO) de la Univer- 
RODRÍGUEZ, I.; FONSECA, G. M. \& ARAMBURÚ, G. Estrés académico en alumnos ingresantes a la carrera de Odontología en la Universidad Nacional de Córdoba, Argentina. Int. J. Odontostomat., 14(4):639-647, 2020.

sidad Nacional de Córdoba en el año 2016. El CINFO posee objetivos claros que pretenden, entre otros, el lograr la adaptación gradual y positiva al nuevo contexto educativo (el universitario), reflexionar acerca de su elección vocacional y adquirir los conocimientos básicos indispensables que lo habilitan para cursar los restantes ciclos de la Carrera (https:// www.odo.unc.edu.ar/academico/alumnos/portal-delingresante). Los estudiantes fueron invitados a participar voluntariamente de este estudio y aquellos que consintieron de participar, fueron convocados a una reunión explicativa donde los investigadores proveyeron toda la información necesaria, así como también los alcances y criterios de inclusión y exclusión. Fueron incluidos alumnos mayores de 18 años y de ambos sexos, excluyéndose aquellos que se encontraron al momento del estudio bajo tratamiento médico por afecciones psiquiátricas (depresión, ansiedad etc.) y/o cualquier tipo de afección médica que involucrara la administración de medicamentos, para evitar sesgos metodológicos en cuanto a la percepción por parte del alumno de síntomas o reacciones que sean propias de su patología de base o propias de la medicación que reciba y así, confundir los síntomas con que se manifiesta el estrés. Fueron excluidos también alumnos recursantes, considerando que habiendo experimentado con anterioridad las diversas situaciones que se presentan en durante el cursado del CINFO, los mismos pueden haber desarrollado estrategias de afrontamiento al estrés.

Tomando en cuenta los criterios de inclusión y exclusión, la muestra de estudio estuvo finalmente conformada por un $n=291$ estudiantes, número que superó el mínimo determinado estadísticamente para el cálculo del tamaño muestral teniendo en cuenta la población general con un nivel de confianza del $95 \%$ :

$$
\mathrm{n}=\frac{N \cdot Z^{2} \cdot p \cdot(1-p)}{(N-1) \cdot \mathrm{e}^{2}+Z^{2} \cdot p \cdot(1-p)}
$$

Técnica e Instrumentos: La recolección de la información se realizó a través de un cuestionario autoadministrado entregado a cada participante durante la actividad teórico-práctica previa al primer examen parcial, considerando esta última, como un estresor importante. El cuestionario utilizado fue el Inventario Sisco (Sistémico Cognoscitivista) del Estrés Académico (Barraza Macías 2007a). El grado de confiabilidad estimado correspondió a 0,90 de Alfa de Cronbach. Dicho cuestionario tuvo un ítem de filtro, en términos dicotómicos (sí / no), que permitió determinar si el encuestado era candidato o no a contestar el cuestionario; un ítem con escalamiento tipo Lickert de cinco valores numéricos (del 1 al 5 , donde 1 es poco y 5 es mucho) para identificar el nivel de intensidad del estrés; diez ítems con escalamiento tipo Lickert de cinco categorías (nunca / rara vez /alguna vez / casi siempre / siempre) para identificar la frecuencia con la que las demandas del entorno fueron valoradas como estresores; y dieciséis ítems con escalamiento tipo Lickert de cinco categorías (nunca / rara vez / alguna vez / casi siempre / siempre) para identificar la frecuencia con que se presentaron los síntomas o reacciones al estímulo estresor. Dichas reacciones fueron divididas en físicas, psicológicas y comportamentales. Por último, el cuestionario contó con nueve ítems con escalamiento tipo Lickert de cinco categorías (nunca / rara vez / alguna vez / casi siempre / siempre) para identificar la frecuencia de uso de estrategias de afrontamiento. Cabe destacar, que este cuestionario fue diseñado específicamente para la evaluación del estrés académico autopercibido en estudiantes universitarios. Como clave de corrección, no fue incorporado a este estudio ningún cuestionario si la pregunta número uno, que es la de filtro, fue contestada con un "NO", aunque el encuestado haya llenado el resto del mismo (Barraza Macías, 2007b).

La interpretación del cuestionario puede hacerse de dos maneras: simple y desglosada (Barraza Macías, 2008a,b), a través del baremo que propone el autor (Tabla I).

Tabla I. Baremo propuesto por Barraza Macías, $2007^{a}$.

\begin{tabular}{cccccc}
\hline \multicolumn{5}{c}{ Intensidad } & \multicolumn{2}{c}{ BAREMO } & \multicolumn{1}{c}{ Intensidad } \\
\hline \multicolumn{2}{c}{ (Interpretación simple) } & (Interpretación & desglosada) & (Pregunta 2) & Nivel \\
Rango & Nivel & Rango & Nivel & Rango & Baja \\
\hline $0-33 \%$ & Leve & 0 a $25 \%$ & Rara vez & O a $20 \%$ & Medianamente baja \\
$34-66 \%$ & Moderado & $26 \%$ a $50 \%$ & Algunas veces & $21 \%$ a $40 \%$ & Media \\
$67-100 \%$ & Profundo & $51 \%$ a $75 \%$ & Casi siempre & $41 \%$ a $60 \%$ & Medianamente alta \\
& & $76 \%$ a $100 \%$ & Siempre & $61 \%$ a $80 \%$ & Alta \\
\hline
\end{tabular}


Interpretación simple: Para este tipo de interpretación es necesario obtener el índice general; el autor recomienda seguir los siguientes pasos:

Se toman para el análisis únicamente los ítems de las preguntas tres (estresores), cuatro (síntomas) y cinco (estrategias de afrontamiento); la opción: otra (especifique), se tomará en cuenta si como mínimo el $10 \%$ de los encuestados llenó esa opción con estresores específicos del ámbito académico.

A cada respuesta de la escala se le asigna los siguientes valores: 0 para nunca, 1 para rara vez, 2 para algunas veces, 3 para casi siempre y 4 para siempre.

Se obtiene la media por cada ítem y posteriormente se obtiene la media general.

Se transforma la media en porcentaje, sea a través de regla de tres simple o multiplicando la media obtenida por 25 .

Una vez obtenido el porcentaje se interpreta con el baremo de intensidad para interpretación simple.

Interpretación desglosada: Para este tipo de interpretación el autor recomienda seguir los siguientes pasos:

Se toman para el análisis únicamente los ítems de las preguntas tres (estresores), cuatro (síntomas) y cinco (estrategias de afrontamiento); la opción: otra (especifique), se tomará en cuenta si como mínimo el $10 \%$ de los encuestados llenó esa opción con estresores específicos del ámbito académico.

A cada respuesta de la escala se le asigna los siguientes valores: 0 para nunca, 1 para rara vez, 2 para algunas veces, 3 para casi siempre y 4 para siempre.

Se obtiene la media por cada ítem, por cada dimensión y por la variable, en lo general.

Se transforma la media en porcentaje, sea a través de regla de tres simple o multiplicando la media obtenida por 25 .

Una vez obtenido el porcentaje se interpreta con el baremo de frecuencia para interpretación desglosada.
En el caso específico de la pregunta número dos (nivel de preocupación o nerviosismo) se obtiene el porcentaje con el procedimiento ya señalado y posteriormente se interpreta con el baremo de intensidad para esta pregunta.

Análisis estadístico: Se realizó un análisis descriptivo de los estresores que caracterizaron al estrés académico autopercibido, de los síntomas asociados al mismo (reacciones físicas, comportamentales y psicológicas) y de las estrategias de afrontamiento utilizadas por los estudiantes. Además, se realizó Análisis de Correlación de Pearson entre las tres dimensiones (Estresores; Reacciones; Estrategias), para lo cual se evaluaron los baremos propuestos por el autor del inventario SISCO. Posteriormente se llevó a cabo un Análisis de Correspondencias Múltiples (ACM) considerando todos los aspectos que conformaron cada dimensión para determinar el grado de asociación entre ellos. Mediante análisis de la varianza (ANOVA), se evaluó si la edad y el sexo de los encuestados fueron factores que pudieran asociarse a un mayor o menor estrés académico, a las reacciones o respuestas físicas y psicológicas y a las estrategias de afrontamiento. En todos los casos se fijó como nivel de significación estadística una p<0,05. Para el análisis estadístico se utilizó el software IBM SPSS Statistics v. 23.0.

Consideraciones éticas: Esta investigación fue evaluada por el Comité Institucional de Ética en Investigaciones en salud (CIEIS). La participación de los estudiantes en dicho estudio fue voluntaria. La aplicación del instrumento se realizó de manera colectiva en la sala de teórico-prácticos de la asignatura Introducción a la Odontología, previa autorización de las autoridades de la Facultad de Odontología de la Universidad Nacional de Córdoba. Todos los estudiantes dieron su consentimiento a la participación de manera previa a la recolección de datos (encuesta).

\section{RESULTADOS}

La muestra estudiada $(n=291)$, estuvo conformada por 228 mujeres (78 \%) y 63 varones $(21,6 \%)$, donde la media de edad fue de 19 años. El $98 \%$ de la población era soltera. En cuanto al lugar de procedencia, el 58,7 \% de los estudiantes fueron de Córdoba (capital e interior), mientras que el restante 41,3 $\%$ fueron oriundos de otras provincias o del exterior del país. 
Determinación del nivel de estrés académico autopercibido. Según los datos obtenidos, el $98,3 \%$ de los alumnos encuestados respondió que presentaba estrés académico, mientras que un 1,7\% manifestó no presentarlo. Por este motivo los cinco alumnos que contestaron "no" a la pregunta de filtro número 1 , no fueron tenidos en cuenta para análisis posteriores.

El nivel de preocupación (pregunta 2 del cuestionario) que presentaron los alumnos fue de 3,74. Este valor correspondió al $74,8 \%$ al ser interpretado con el baremo de intensidad, lo que indicó un nivel de estrés académico concordante con la categoría Medianamente alta.

El $88,81 \%$ de los participantes se inclinó por las categorías de intensidad más altas de estrés, es decir entre la 3 y la 5 , mientras que el $11,19 \%$ lo hizo dentro del nivel 1 y 2 . El $29,37 \%$ del total de los participantes marcó el máximo nivel de preocupación (nivel 5) (Fig. 1).

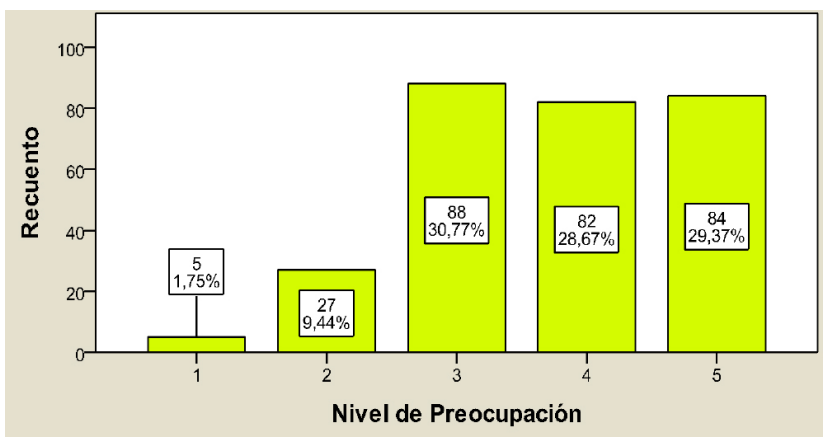

Fig. 1. Histograma de casos según nivel de preocupación.

Tabla III. Tabla de frecuencias de reacciones físicas, psíquicas y comportamentales.

\begin{tabular}{llccc}
\hline & Reacciones & Media & DE & $\%$ \\
\hline Físicas & Fatiga crónica & 2,3 & 1,1 & 57,5 \\
& Somnolencia & 2,3 & 1,2 & 57,3 \\
& Trastornos del sueño & 2 & 1,2 & 49,2 \\
& Dolores de cabeza & 1,9 & 1,2 & 48,6 \\
& Rascarse y morderse las uñas & 1,8 & 1,4 & 44,9 \\
Psíquicas & Problemas de digestión & 1,2 & 1,2 & 29,8 \\
& Ansiedad, angustia & 2,3 & 1,2 & 57,6 \\
& Inquietud & 2,2 & 1,2 & 55,1 \\
& Problemas de concentración & 2,2 & 1,1 & 54,7 \\
& Depresión y tristeza & 2,1 & 1,3 & 53,2 \\
Comportamental & Agresividad & 1,3 & 1,3 & 33,6 \\
& Aumento o reducción en & 2,2 & 1,3 & 55,9 \\
& consumo de alimentos & & & \\
& Desgano & 1,8 & 1 & 44,6 \\
& Conflictos y tendencia a & 1 & 1 & 25,8 \\
& Aiślamiento & 1 & 1,1 & 25,7 \\
\hline
\end{tabular}

Tabla II: Niveles de estresores: Desviación estándar (DE).

\begin{tabular}{lccc}
\hline \multicolumn{1}{c}{ Estresor } & Media & $\mathrm{DE}$ & $\%$ \\
\hline Evaluaciones & 2,7 & 1,07 & 67,4 \\
Sobrecargas de tareas & 2,53 & 0,94 & 63,4 \\
No entender los temas de las clases & 2,37 & 1 & 59,2 \\
Tiempo limitado para trabajar & 2,16 & 1,19 & 54,1 \\
Participación en clases & 1,63 & 1,22 & 40,6 \\
Tipo de trabajo que piden los Prof. & 1,53 & 1,03 & 38,4 \\
Personalidad y carácter del Prof. & 1,26 & 0,97 & 31,6 \\
Problemas con horarios de clases & 1,24 & 1,2 & 31,1 \\
Competencia c/compañeros & 0,75 & 0,93 & 18,7 \\
Otra & 0,42 & 1,17 & 10,5 \\
\hline
\end{tabular}

\section{Caracterización del estrés académico}

Identificación de situaciones (estresores) que generan estrés académico. El nivel de intensidad de la dimensión "Estresores" fue Moderado (41,5 \%) según la interpretación del baremo propuesto por el autor. Las situaciones que con más frecuencia generaron estrés académico a los participantes fueron las evaluaciones de los profesores que con un valor de $67,4 \%$ se ubicó en la categoría de Profundo, la sobrecarga de tareas quedó como segunda situación más frecuente con un valor de $63,4 \%$ con categoría de Moderado (Tabla II).

Identificación de los síntomas (reacciones) que caracterizan al estrés académico. En cuanto a los síntomas generados por el estrés académico, el nivel de intensidad de la dimensión fue Moderado según la interpretación del baremo, con un porcentaje de $46,2 \%$, mientras que en la interpretación desglosada el valor obtenido quedó incluido en la categoría Algunas veces.

Las reacciones físicas más frecuentes fueron fatiga crónica $(57,5 \%)$ y somnolencia $(57,3 \%)$, ambas dentro de un rango de intensidad Moderado, siendo las menos frecuentes los problemas digestivos, en categoría Leve. Las reacciones psicológicas más frecuentes fueron la ansiedad, angustia e inquietud, ambas con rango de intensidad Moderado, mientras que la menos frecuente fue la agresividad que categorizó como Leve; por último las reacciones comportamentales más frecuentes fueron, el aumento o reducción del consumo de alimentos con frecuencia del $55,9 \%$ y el desgano con una frecuencia de 44,6\%, ambas en rango de intensidad Moderado, y la que obtuvo menor frecuencia fue conflictos y tendencia a polemizar o discutir y aislamiento de los demás ambas en categoría Leve (Tabla III). 
Identificación las estrategias de afrontamiento con que cuentan los alumnos. El nivel de intensidad general de la dimensión "Estrategias de Afrontamiento" fue Moderado (50,7 \%). Al realizar un análisis detallado de cada una de las estrategias de afrontamiento se verificó que la estrategia concentrarse en resolver la situación fue la que mayor porcentaje consiguió un $72,1 \%$ en un rango de nivel Profundo, seguida por las estrategias tratar de obtener lo positivo $(63,4 \%)$ y elaboración de plan y ejecución de tareas $(60,7 \%)$. La estrategia que menor frecuencia tuvo fue elogios a sí mismo (Tabla IV).

Tabla IV Estrategias de afrontamiento.

\begin{tabular}{lcc}
\hline \multicolumn{1}{c}{ Estrategia } & Media & $\mathrm{DE}$ \\
\hline Concentrarse en resolver la situación & 2,88 & 1 \\
Tratar de obtener lo positivo & 2,54 & 1,2 \\
Elaboración de plan y ejecución de tareas & 2,43 & 1,1 \\
Habilidad asertiva & 2,06 & 1,3 \\
Ventilación y confidencias & 2,02 & 1,3 \\
Búsqueda de información & 1,76 & 1,3 \\
La religiosidad & 1,39 & 1,4 \\
Elogios a sí mismo & 1,13 & 1,1
\end{tabular}

Luego de la descripción de cada dimensión se realizó la correlación entre variables. Considerando los valores de intensidad porcentual medios de cada dimensión, se verificó mediante el test de Correlación de Pearson una muy significativa correlación entre ESTRESORES y REACCIONES $(p<0,001)$ y una significativa correlación entre ESTRESORES y ESTRATEGIAS ( $p=0,034)$; las dimensiones REACCIONES y ESTRATEGIAS no se correlacionaron significativamente $(p=0,125)$ (Tabla V).

Tabla V. Correlación entre dimensiones.

\begin{tabular}{lccccl}
\hline \multicolumn{1}{c}{ Dimensión } & \multicolumn{2}{c}{ Femenino } & Masculino & $\begin{array}{l}\text { ANOVA } \\
(\mathrm{p} \text {-valor) }\end{array}$ \\
& $\mathrm{n}$ & Media & $\mathrm{N}$ & Media & \\
\hline ESTRESORES & 227 & 43,1 & 59 & 35,3 & $1,18 \times 10^{4} ; \mathrm{p}<0,05$ \\
REACCIONES & 226 & 49,2 & 59 & 35,0 & $9,89 \times 10^{-9} ; \mathrm{p}<0,05$ \\
ESTRATEGIAS & 226 & 50,2 & 59 & 52,4 & 0,$346 ; \mathrm{p}>0,05$ \\
\hline
\end{tabular}

Las diferencias entre mujeres y hombres, según los datos obtenidos mediante el análisis de la varianza (ANOVA), se presentaron en la dimensión REACCIONES, en donde las mujeres registraron valores significativamente más altos que los hombres. También fueron significativas las diferencias en la dimensión ESTRESORES, mientras que en la dimensión ESTRATEGIAS, las puntuaciones generales no fueron significativas (Tabla VI).
Tabla VI. ANOVA para factor sexo.

\begin{tabular}{|c|c|c|c|}
\hline Dimensión & $\mathrm{n}$ & Media & $\begin{array}{l}\text { Correlación } \\
\text { de Pearson }\end{array}$ \\
\hline ESTRESORES & 286 & 41,5 & 0,575 \\
\hline REACCIONES & 285 & 46,2 & \\
\hline Dimensión & $\mathrm{n}$ & Media & $\begin{array}{l}\text { Correlación } \\
\text { de Pearson }\end{array}$ \\
\hline ESTRESORES & 286 & 41,5 & 0,126 \\
\hline ESTRATEGIAS & 285 & 50,7 & \\
\hline Dimensión & $\mathrm{n}$ & Media & $\begin{array}{l}\text { Correlación } \\
\text { de Pearson }\end{array}$ \\
\hline REACCIONES & 285 & 46,2 & 0,091 \\
\hline ESTRATEGIAS & 285 & 50,7 & \\
\hline
\end{tabular}

La Figura 2 muestra las distribuciones de valores de las dos dimensiones en las cuales se diferenciaron las mujeres de los hombres. Los centroides correspondientes a cada sexo coinciden con las medias de cada grupo. Se observa claramente el desplazamiento marcado en mujeres con mayores manifestaciones en estresores y reacciones.

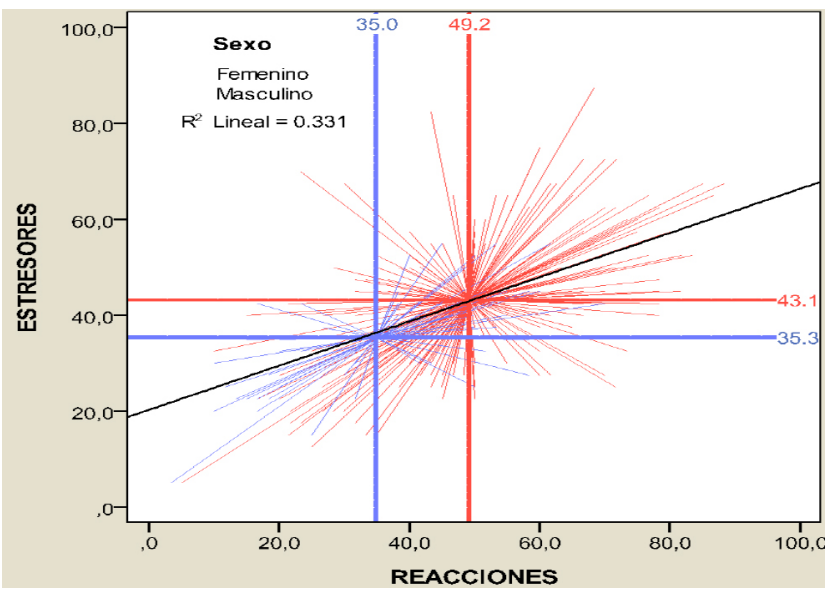

Fig. 2. Diagrama de dispersión: Nivel general de ESTRESORES vs nivel general de REACCIONES según sexo.

Otro resultado de interés fue la asociación de mujeres a mayores niveles de reacciones físicas y psicológicas respecto a los valores registrados en hombres (Fig. 3).

Fue dentro de la dimensión "estresores" donde se apreciaron las mayores diferencias entre grupos etarios, a menor edad mayor nivel de los estresores $(p<0,05)$. Al considerar la dimensión reacciones, las diferencias fueron menores, pero también significativas $(p<0,05)$, datos obtenidos mediante el análisis de la varianza. Respecto a la dimensión estrategias de aprendizaje, la edad no resultó ser un factor asociado (Tabla VII). 


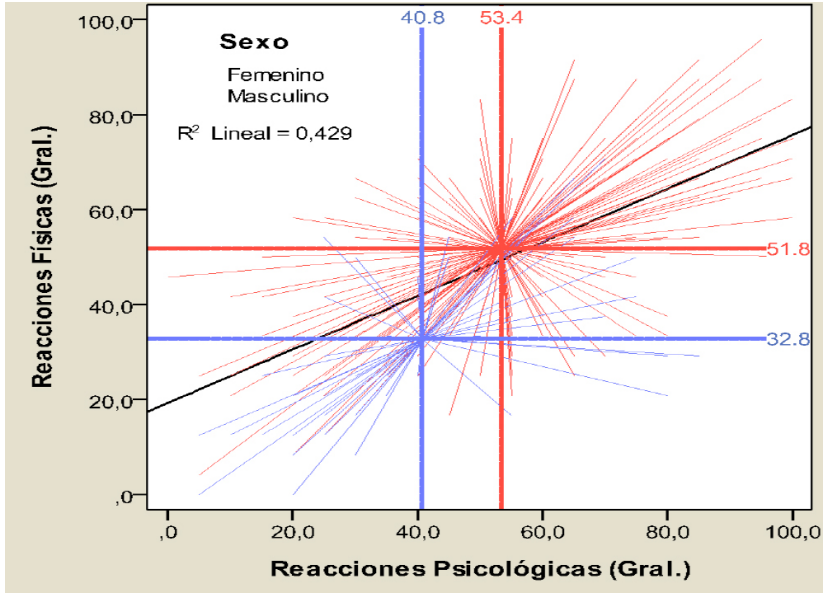

Fig. 3. Diagrama de dispersión: Nivel general.

Tabla VII. Contrastes entre grupos etarios según dimensión. Medias y nivel de significación de la prueba estadístico.

\begin{tabular}{ccccc}
\hline Dimensión & $\begin{array}{c}18 \text { años } \\
(\mathrm{n}=187)\end{array}$ & $\begin{array}{c}19 \text { años } \\
(\mathrm{n}=50)\end{array}$ & $\begin{array}{c}20 \text { o más A. } \\
(\mathrm{n}=49)\end{array}$ & $\begin{array}{c}\text { ANOV } \\
(\mathrm{p} \text {-valc }\end{array}$ \\
\hline ESTRESORES & 41,71 & 37,85 & 37,35 & 0,$004 ;$ \\
REACCIONES & 47,38 & 43,37 & 41,42 & 0,$044 ;$ \\
ESTRATEGIAS & 51,4 & 51,07 & 49,36 & 0,$909 ;$
\end{tabular}

Para poder precisar el grupo etario en el que se observaba esta relación significativa entre variables se realizó test de Tukey. En cuanto a estresores, los alumnos de 18 años se diferenciaron significativamente de los más grandes (20 o más años) $(p=0,049)$, en tanto que el grupo de 19 años, con niveles de estresores intermedios no se diferenció significativamente ni de los más jóvenes ni de los de mayor edad $(p>0,05)$ (Tabla VIII).

Tabla VIII. Test de Tukey. Relación estresores-edad.

\begin{tabular}{lccc}
\hline (I) G Etario & $(\mathrm{J})$ G Etario & $\begin{array}{c}\text { Diferencia } \\
\text { de medias } \\
(\mathrm{I}-\mathrm{J})\end{array}$ & Sig. \\
\hline 18 años & 19 años & 5,28 & 0,082 \\
19 años & 20 o más & $\left(^{*}\right) 5,78$ & 0,049 \\
& 18 ã̃os & $-5,28$ & 0,082 \\
20 o más & 20 o más & 0,50 & 0,998 \\
años & 18 ãños & $\left(^{*}\right)-5,78$ & 0,049 \\
\hline
\end{tabular}

En lo referido a la dimensión "Reacciones" pudo observarse que el grupo etario que presentó mayor frecuencia de reacciones fue el grupo de 18 años con $47,38 \%$ mientras que el grupo con menor frecuencia de reacciones fue el grupo etario de 20 años o más $(41,42 \%)$ (Fig. 4).

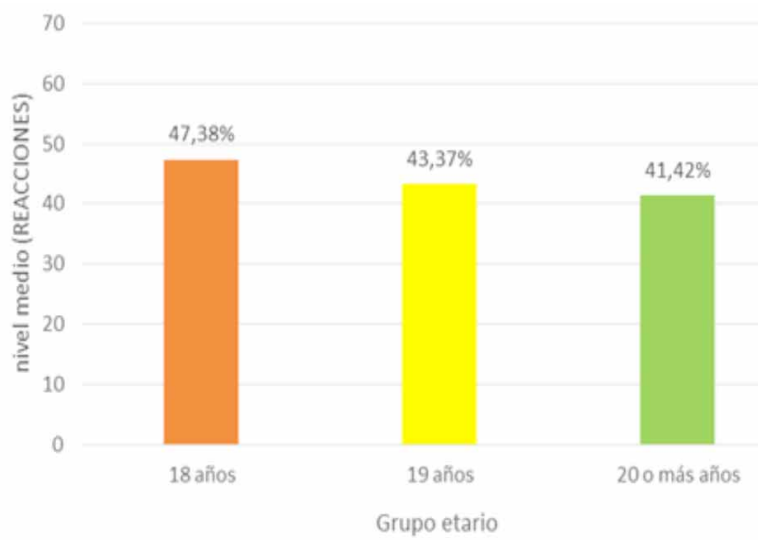

Fig. 4. Nivel medio de puntaje de la dimensión REACCIONES según grupo etario.

Con el test de Tukey se visualizó que los alumnos de 18 años registraron los valores más elevados de reacciones y se diferenciaron significativamente del grupo 20 o más años, el cual registró los valores más bajos $(p=0,047)$ (Tabla IX).

Tabla IX. Test de Tukey. Relación reacciones-edad.

\begin{tabular}{|c|c|c|c|}
\hline (I) G Etario & (J) G Etario & $\begin{array}{c}\text { Diferencia de } \\
\text { medias (I-J) }\end{array}$ & Sig. \\
\hline \multirow[t]{2}{*}{18 años } & 19 años & 5,21 & 0,245 \\
\hline & 20 o más años & $\left(^{*}\right) 7,15$ & 0,047 \\
\hline \multirow[t]{2}{*}{19 años } & 18 años & $-5,21$ & 0,245 \\
\hline & 20 o más años & 1,94 & 0,943 \\
\hline \multirow[t]{2}{*}{20 o más años } & 18 años & $\left(^{*}\right)-7,15$ & 0,047 \\
\hline & 19 años & $-1,94$ & 0,943 \\
\hline
\end{tabular}

\section{DISCUSION}

El estrés académico fue un fenómeno generalizado en la muestra estudiada ya que el $98,3 \%$, presentó estrés académico autopercibido, con un grado medianamente alto. Los estresores más frecuentes fueron las evaluaciones y la sobrecarga de tareas, y menos frecuente la competencia con los compañeros, datos que coinciden con los reportados por Barraza Macías (2009), Díaz Cárdenas et al. y García-Ros et al. (2012). En contraposición, para Castillo Pimienta et al., la sobrecarga de tareas es lo más frecuente, siguiendo en orden de frecuencia, la falta de tiempo para realizar las tareas académicas, y las evaluaciones.

En cuanto a los síntomas, los más frecuentes en este estudio fueron la fatiga crónica, la ansiedad, la somnolencia, y el aumento o reducción de consu- 
mo de alimentos, datos similares a los obtenidos por Díaz Cárdenas et al., quienes reportan que la somnolencia y el aumento del consumo de alimentos son los más frecuentes mientras que los problemas de digestión, agresividad, tendencia a polemizar y aislamiento son los menos frecuentes. Más recurrentes en el grupo etario de 18 años y en mujeres fueron las dimensiones estresores y reacciones, dato coincidente también con Díaz Cárdenas et al.

La estrategia de afrontamiento más usada por los alumnos en la muestra fue la concentración en resolver la situación, no obteniendo para esta dimensión, asociación ni con el sexo ni con la edad, datos coincidentes con lo informado por Barraza Macías (2009). Para Díaz Cárdenas et al., la religiosidad es la estrategia más frecuente, datos opuestos a este estudio ya que esa estrategia estuvo dentro de las menos frecuentes junto con elogios a sí mismo. El componente estrategias de afrontamiento fue el que se presentó con mayor frecuencia entre los estudiantes, mientras que el componente síntomas se presentó con menor frecuencia, datos que coinciden con los reportados por Barraza Macías (2007b).

Una probable explicación del porqué los estudiantes de edades más bajas fueron los más estresados en este estudio (niveles de estresores y reacciones más marcados), coincidente con lo informado por la mayoría de los autores (Barraza Macías, 2005, 2008a,b; 2009; Berrío García \& Mazo Zea; Cabanach et al.; Quito Calle et al.), podría deberse a que los cambios fisiológicos, afectivos y cognitivos que ocurren con la maduración, conducen al alumno a encontrar con los años, nuevas estrategias para afrontar las situaciones de estrés.

Sería sumamente valioso seguir estudiando todas estas variables en estudios longitudinales, para determinar si existen y en qué grado, estrategias de adaptación a lo largo del cursado de la carrera. Debido a que ya ha sido demostrada la utilización de diferentes drogas -legales o ilegales- para paliar los efectos desagradables del estrés, se propone continuar esta línea de investigación para evaluar en esta muestra el probable consumo de sustancias psicoactivas relacionándolas como variables influyentes en el rendimiento académico. Habiendo demostrado que el estrés académico es un factor muy trascendente en la vida del estudiante universitario, sería relevante ajustar ciertos parámetros para evitar el agobio y la sobrecarga de tareas, así como también fortalecer los recursos personales y pedagógicos (estrategias de afron- tamiento) para que puedan afrontar dichas situaciones de la mejor manera. Es por ello, que se requieren de nuevas estrategias incluso hasta institucionales, para lograr que los alumnos puedan enfrentar con éxito las situaciones académicas que los aquejan.

\section{CONCLUSIONES}

Se determinó el nivel de estrés académico autopercibido en un $98,3 \%$ de los estudiantes muestreados, donde el $88,81 \%$ se inclinó por las categorías de intensidad más altas de estrés. Las variables más frecuentes detectadas fueron las evaluaciones y la sobrecarga de tareas como estresores, y la fatiga crónica, la ansiedad, la somnolencia, y el aumento o reducción de consumo de alimentos como síntomas. Existió correlación significativa entre las dimensiones Estresores y Reacciones $(p<0,001)$ y entre Estresores y Estrategias $(p=0,034)$. La estrategia de afrontamiento más usada fue la concentración en resolver la situación, no presentando asociación ni con el sexo ni con la edad. Se sugiere evaluar la relación de estrés académico con el probable consumo de sustancias psicoactivas relacionándolas como variables influyentes en su rendimiento.

\section{AGRADECIMIENTOS}

Los autores desean agradecer especialmente al Dr. Ariel Abeldaño, de la Universidad de la Sierra Sur, Oxaca (México), por su aporte al análisis estadístico de este trabajo.

RODRÍGUEZ, I.; FONSECA, G. M. \& ARAMBURÚ, G. Academic stress of freshmen students at the Faculty of Dentistry, Universidad Nacional de Cordoba, Argentina). Int. J. Odontostomat. 14(4):639-647, 2020.

ABSTRACT: Stress is a state of instability and emotional anguish that complicates the ability to focus and function effectively on a routine basis. In educational science, it is called academic stress encompassing three dimensions: stressors, general health reactions, and stress regulators. Several studies have indicated that health career students may be exposed to higher stress levels than the general population. The aim of this study was to evaluate the selfperceived academic stress in freshmen students at the Faculty of Dentistry, Universidad Nacional de Cordoba (Cordoba, Argentina). A descriptive study was carried out, where the sample consisted of 291 students. The information 
was collected through the self-administered questionnaire Sisco Inventory of Academic Stress (Barraza Macias, 2007). Data analysis was carried out using specific scales, and for the association between variables, Pearson and Anova tests were used, setting the level of a statistical significance at $p<0.05$. A significant majority of the students (98.3\%) presented academic stress, with $88.81 \%$ of participants leaning towards the highest intensity categories of stress. A very significant correlation was identified between the Stressors and Reactions dimensions $(p<0.001)$ and between Stressors and Strategies $(p=0.034)$. The identification of academic stress, its characterization and prevention of potential habits or use of medication often considered unhealthy, will promote the development of strategies for students, in order to better face situations encountered in the new environment.

KEY WORDS: self-perceived stress, dental students, stressor, coping mechanisms.

\section{REFERENCIAS BIBLIOGRAFICAS}

Barraza Macías, A. \& Silerio Quiñónez, J. S. El estrés académico en alumnos de educación media superior: un estudio comparativo. INED, 2(7):48-65, 2007.

Barraza Macías, A. Características del estrés académico de los alumnos de educación media superior. INED, 1(4):15-20, 2005.

Barraza Macías, A. Construcción y validación psicométrica del inventario del estrés de examen. Investig. Educ. Duranguense, (9):33-45, 2008a.

Barraza Macías, A. El estrés académico en alumnos de maestría y sus variables moduladoras: un diseño de diferencia de grupos. Av. Psicol. Latinoam., 2(26):270-89, 2008b.

Barraza Macías, A. El inventario SISCO del estrés académico. INED, 2(7):89-93, 2007a.

Barraza Macías, A. Estrés académico y burnout estudiantil. Análisis de su relación en alumnos de licenciatura. Psicogente, 12(22):272-83, 2009.

Barraza Macías, A. Propiedades Psicométricas del Inventario SISCO del Estrés Académico. Rev. Psicol. Cient., 2007b. Disponible en: https://www.psicologiacientifica.com/sisco-propiedadespsicometricas/

Berrío García, N. \& Mazo Zea, R. Estrés Académico. Rev. Psicol. Univ. Antioq., 3(2):65-82, 2011.

Cabanach, R. G.; Souto Gestal, A. \& Franco, V. Escala de Estresores Académicos para la evaluación de los estresores académicos en estudiantes universitarios. Rev. Iberoam. Psicol. Salud, 7(2):41-50, 2016.

Castillo Pimienta, C.; Chacón de la Cruz, T. \& Díaz-Véliz, G. Ansiedad y fuentes de estrés académico en estudiantes de carreras de la salud. Investig. Educ. Med., 5(20):230-7, 2016.

Díaz Cárdenas, S.; Arrieta Vergara, K. \& González Martínez, F. Estrés académico y funcionalidad familiar en estudiantes de odontología. Salud Uninorte, 30(2):121-32, 2014.

García-Ros, R.; Pérez-González, F.; Pérez-Blasco, J. \& Natividad, L. A. Evaluación del estrés académico en estudiantes de nueva incorporación a la universidad. Rev. Latinoam. Psicol., 44(2):14354, 2012.

Marín Laredo, M. M.; Álvarez Huante, C. G.; Lizalde Hernández, A.; Anguiano Morán, A. \& Lemus Loeza B. M. Estrés académico en estudiantes. El caso de la Facultad de Enfermería de la Universidad Michoacana. Rev. Iberoam. Prod. Acad. Gest. Educ., 1(1):1-17, 2014.

Martín Monzón, I. M. Estrés académico en estudiantes universitarios. Apunt. Psicol., 1(25):87-99, 2007.

Nadal, R. \& Armario, A. Mecanismos de susceptibilidad al estrés. Hipertens. Riesgo Vasc., 27(3):117-24, 2010.

Qamar, K.; Khan, N. S. \& Kiani, M. R. B. Factors associated with stress among medical students. J. Pak. Med. Assoc., 65(7):753$5,2015$.

Quito Calle, J. V.; Tamayo Piedra, M.; Buñay Barahona, D. P. \& Neira Cárdenas, O. S. Estrés académico en estudiantes de tercero de bachillerato de unidades educativas particulares del Ecuador. Rev. Electron. Psicol. Iztacala, 20(3):253-76, 2017.

Rodríguez García, B.; Labrador Ortega, M. \& Blanco Montagut, L. E. Estresores académicos percibidos por estudiantes pertenecientes al Grado en Enfermería de la escuela de Enfermería y Fisioterapia de la Universidad de Salamanca. Rev. Enferm. CyL, 8(2):23-32, 2016.

Dirección para correspondencia:

Dr. Gabriel M. Fonseca

Centro de Investigación en Odontología Legal y Forense

Facultad de Odontología

Universidad de La Frontera

Francisco Salazar 01145

Temuco

CHILE

Email: gabriel.fonseca@ufrontera.cl

Recibido : 30-03-2020

Aceptado: 24-06-2020 